\title{
Endocrine dysfunctions during treatment of immune-checkpoint inhibitors
}

\author{
Hidefumi Inaba ${ }^{1^{*}}$, Hiroyuki Ariyasu ${ }^{1}$, Hisako Okuhira', Yuki Yamamoto ${ }^{2}$, Hiroaki Akamatsu ${ }^{3}$, \\ Masahiro Katsuda ${ }^{4}$, Masatoshi Jinnin', Isao Hara', Takashi Akamizu' \\ ${ }^{1}$ The First Department of Medicine, Wakayama Medical University, Wakayama, Japan \\ ${ }^{2}$ Department of Dermatology, Wakayama Medical University, Wakayama, Japan \\ ${ }^{3}$ Department of Respiratory Medicine and Clinical Oncology, Wakayama Medical University, Wakayama, Japan \\ ${ }^{4}$ Second Department of Surgery, Wakayama Medical University, Wakayama, Japan \\ ${ }^{5}$ Department of Urology, Wakayama Medical University, Wakayama, Japan
}

\begin{abstract}
Immune-checkpoint inhibitors (ICIs) are novel agents directed to various malignant tumors. During ICI therapy, however, immune related adverse effects (irAEs) including endocrine dysfunctions have been reported. Dysfunctions in the pituitary gland and the thyroid gland by ICI are often observed, and those in the adrenal glands and the pancreas are less frequent. Positive correlation of the prevalence of endocrine irAEs to clinical antitumor effectiveness during ICI therapy has been reported. The mechanisms of endocrine irAEs by ICI, however, remain unclear, and optimal prevention, prediction, and treatment of the irAEs are still uncertain. This review describes possible mechanisms involved in ICI-related immunity, and discusses clinical management of endocrine irAEs during ICI therapy.
\end{abstract}

Keywords: PD-1; CTLA-4; immune-checkpoint inhibitors; endocrine organs; irAEs

ARTICLE INFO

Received: April 28, 2020

Accepted: May 25, 2020

Available online: June 7, 2020

*CORRESPONDING AUTHOR

Hidefumi Inaba, The First Department of Medicine, Wakayama Medical University, 811-1, Kimiidera, Wakayama, Japan; inaba@wakayamamed.ac.jp

CITATION

Inaba $\mathrm{H}$, Ariyasu $\mathrm{H}$, Okuhira $\mathrm{H}$, et al. Endocrine dysfunctions during treatment of immune-checkpoint inhibitors. Trends Immunother 2020; 4(1): 18-26.

doi:10.24294/ti.v4.i1.606.

COPYRIGHT

Copyright (C) 2020 by author(s) and EnPress Publisher LLC. This work is licensed under the Creative Commons Attribution-NonCommercial 4.0 International License (CC BY-NC 4.0). http://creativecommons.org/licenses/ by $/ 4.0 /$

\section{Introduction}

Immune-checkpoints play an indispensable role in anti-tumor immunity, anti-infection and autoimmunity ${ }^{[1,2]}$. Their major components include cytotoxic T-lymphocyte associated antigen 4 (CTLA-4), programmed death protein 1 (PD-1), and ligand for PD-1 (PD-L1) (Figure 1, 2). CTLA-4 is located on the surface of activated T-cells, therefore inhibiting binding of CD28 to B7 molecule on antigen presenting cells (APC) ${ }^{[1]}$. CTLA-4 pathway predominantly acts in lymph nodes. PD-1 is majorly expressed on T-cells ${ }^{[2]}$. PD-L1 is usually expressed on tumor cells, T-cells, B-cells, dendritic cells ${ }^{[2]}$. PD-1 pathway is involved with tumor microenvironment. APC or tumor cells present cancer-related antigen with major histocompatibility (MHC)-class I to T-cell receptor (TCR) on cytotoxic $\mathrm{CD}^{+} \mathrm{T}$-cell (Figure 1). Additionally, APC presents cancer-related antigen with MHC-class II to TCR on helper CD4 ${ }^{+}$ T-cell (Figure 2). $\mathrm{CD}^{+}$Th1-T-cells activate $\mathrm{CD} 8^{+} \mathrm{T}$-cells, and $\mathrm{CD} 4^{+} \mathrm{Th} 2-\mathrm{T}$ cells stimulate antibody production. In antitumor immunity, $\mathrm{CD} 4^{+} \mathrm{T}$-cell and $\mathrm{CD} 8^{+} \mathrm{T}$-cell cooperate in the network.

Monoclonal antibodies to immune-checkpoints are referred to as immunecheckpoint inhibitors (ICIs), and are currently considered to be novel promising agents for treatment of malignant tumors ${ }^{[3,4]}$. ICI promotes T-cell mediated cytotoxicity directed to cancer cell antigens. It has been reported to improve prognosis of patients with malignant melanoma, renal cell cancer, non-small cell lung cancer, Hodgkin's lymphoma, and Merkel cell carcinoma. Approximately $20 \%-30 \%$ of patients with malignant melanoma, renal cell cancer, and non-small cell lung cancer were found to be responders of ICI. More than $50 \%$ of patients showed objective responses in the patients with refractory Hodgkin's lymphoma or Merkel cell carcinoma. On the other hand, various adverse events were reported as immune-related adverse 
events (irAEs). IrAEs include dermatological, gastrointestinal, hepatic, neurological, and endocrine disorders $^{[3,4]}$ (Table 1).

In endocrine organs, irAEs in the pituitary gland, the thyroid gland, the parathyroid glands, the adrenal glands, the pancreas (type 1 diabetes mellitus) were reported $^{[5-13]}$. Although ICI activates T-cells through inhibition of immune-checkpoints molecules, the etiology of endocrine irAEs remains unclear. Endocrine dysfunctions are sometimes crucial, and diagnostic procedures in endocrine dysfunctions are often complicated. Routine follow-up including physical examination with endocrinological investigation, as well as image testing is important. This review describes endocrine dysfunction during ICI therapy, and raises possible mechanisms involved with ICI-related immunity, highlighting diagnostic approaches and appropriate clinical management during ICI therapy.

\section{Hypophysitis related to irAE}

The pituitary gland is an endocrine organ central to regulate peripheral endocrine glands, by secreting several pituitary hormones; adrenocorticotrophic

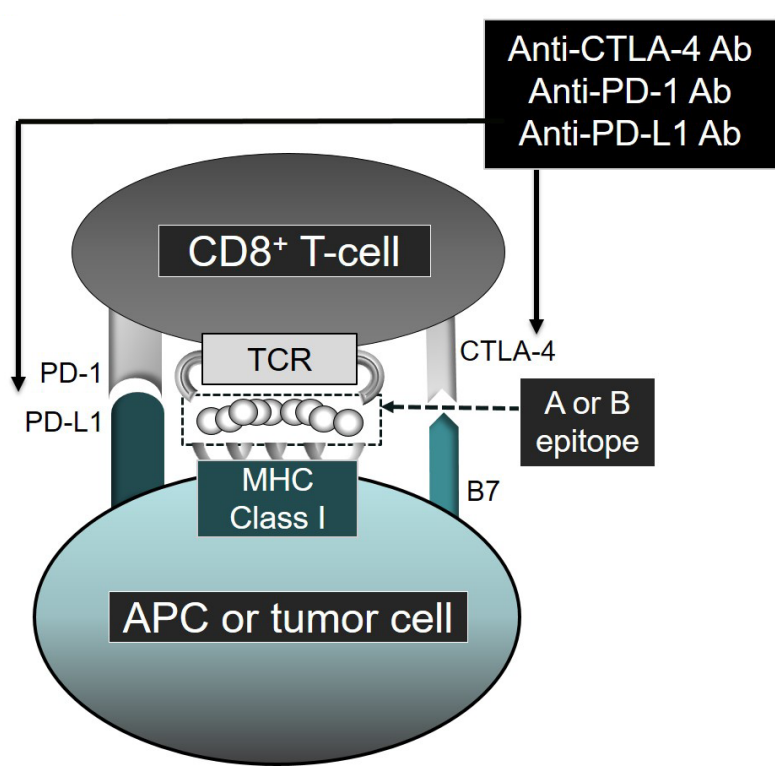

Figure 1. Relation of immune-checkpoints and $\mathrm{CD} 8^{+}$ T-cell is shown. A or B epitopes are presented on the surface of APC or tumor cell with MHC-class I to TCR on $\mathrm{CD}^{+} \mathrm{T}$-cell. Immune-checkpoint inhibitors regulate these immune reaction, and $\mathrm{CD}^{+} \mathrm{T}$-cell-mediated cytotoxic effect is enhanced.

A: tumor antigen, B endocrine organs specific antigens (e.g. thyroglobulin, insulin). APC: antigen presenting cell; TCR: T-cell receptor hormone (ACTH), thyrotropin (TSH), gonadotropin, growth hormone $(\mathrm{GH})$, and prolactin, and antidiuretic hormone $(\mathrm{ADH})$. The estimated prevalence of irAE related pituitary dysfunction is $9.1 \%{ }^{[5]}$, in contrast to idiopathic autoimmune hypophysitis which is only observed in 1 in 9 million individuals ${ }^{[14]}$. Incidence of irAE-related hypophysitis varies $(0.4 \%-17.2 \%)$ in previous reports, the wide range associated with differences in surveillance $\mathrm{e}^{[4,7,15,16]}$. Hypophysitis related to irAE occurs mostly within 6 months. In pituitary dysfunction, the incidence of secondary adrenal insufficiency: 'ACTH insufficiency', secondary hypothyroidism; 'TSH insufficiency', and secondary hypogonadism were $6.1 \%, 7.6 \%$, and $7.5 \%$, respectively ${ }^{[5]}$. The prevalence of $\mathrm{GH}$ insufficiency is not clear. Although rare, central diabetes insipidus due to pituitary posterior lobe dysfunction; 'ADH insufficiency' during ICI therapy was reported recently ${ }^{[17]}$.

Most of hypophysitis related to irAE is induced by anti-CTLA-4 antibody, and anti-PD-1 antibodyinduced hypophysitis is less common $(<1.0 \%)$ $[4,18]$. Hypophysitis related to irAE occurs more often in men than women ${ }^{[15,16]}$. In contrast, female predominance is seen in idiopathic autoimmune hypophysitis ${ }^{[14]}$. Common clinical manifestations in secondary adrenal insufficiency include headaches, fatigue, muscle weakness, and nausea, anorexia, weight loss, and hypotension are often reported ${ }^{[7,15,16]}$. In secondary hypothyroidism, constipation, fatigue, edema, and bradycardia can be seen. Manifestations in secondary hypogonadism and GH insufficiency can be not clear, but may be general fatigue and appetite loss. Notably, ACTH or TSH insufficiency are the most common pituitary hormone abnormalities in hypophysitis related to irAE ${ }^{[15,16]}$.

Secondary adrenal insufficiency is diagnosed with decreased ACTH and cortisol, and severe cases show hypoglycemia, hyponatremia, and hyperkalemia. Secondary hypothyroidism is diagnosed with decreased FT3, FT4, TSH, and sometimes hyponatremia, and elevated CK or cholesterol are seen. Secondary hypogonadism was diagnosed with decreased gonadotropin with decreased sex hormones (estradiol or testosterone). Low levels of GH and insulin-like growth factor-1 (IGF-1) are seen in GH insufficiency. In imaging tests, pituitary enlargement is sometimes seen in hypophysitis related to irAE in pituitary MRI ${ }^{[15,16]}$. Incomplete secretion of $\mathrm{ADH}$ and disappearance of $\mathrm{T} 1$-weighed high signal in posterior lobe in pituitary MRI is observed in central diabetes insipidus ${ }^{[17]}$. In order to precisely diagnose, pituitary provocation tests is often recommended. ACTH insufficiency is usually permanent, on the other 

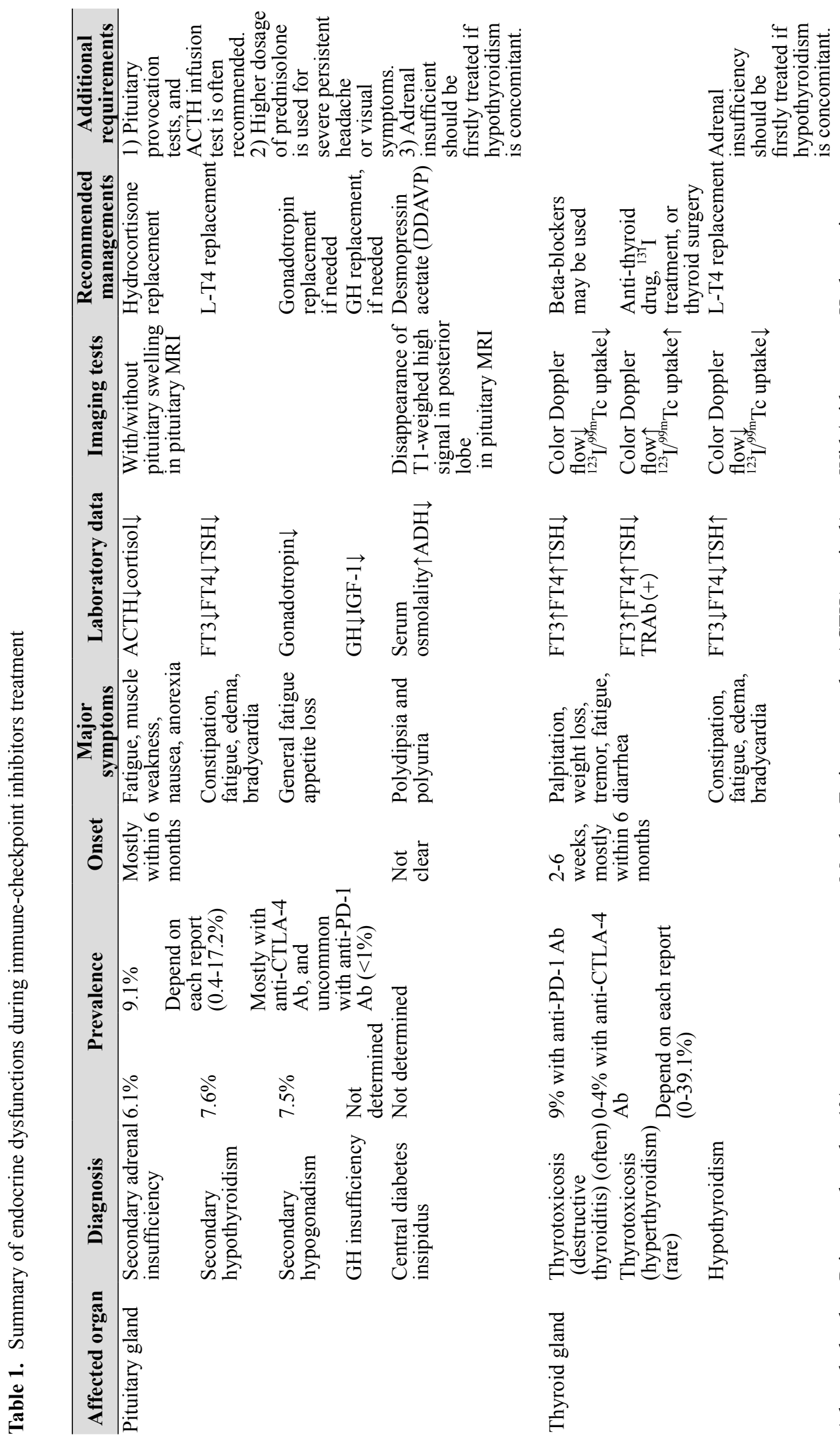

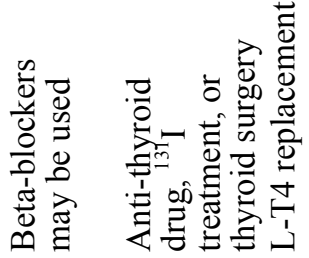

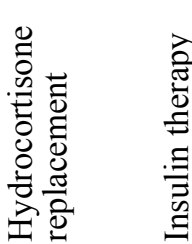

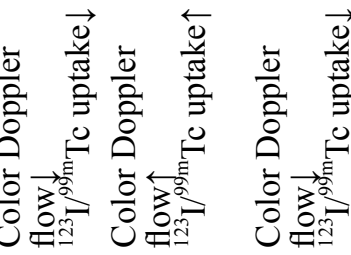

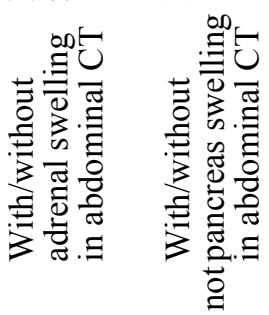

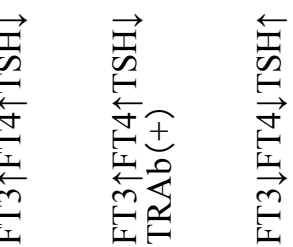

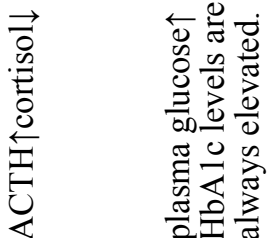

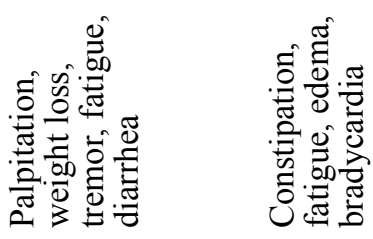

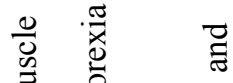

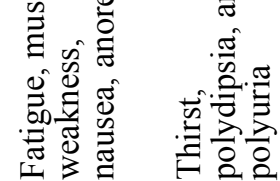

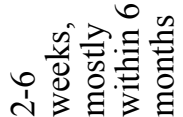

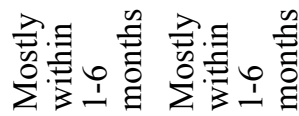

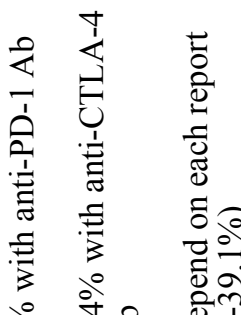

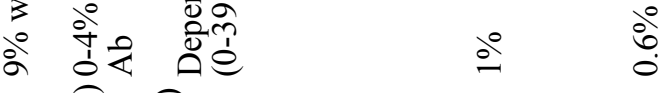

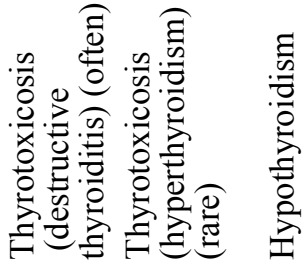

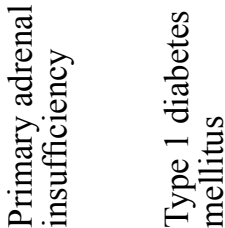

를

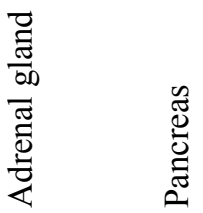


hand, secondary hypothyroidism/hypogonadism may recover. Positivity of anti-pituitary antibody may be helpful for diagnosis of hypophysitis related to irAE ${ }^{[18,19]}$. As predictive factors for hypophysitis related to irAE, eosinophilia, elevation of ESR/CRP/ $\mathrm{LDH}$, and hyponatremia can be useful ${ }^{[9]}$.

In monitoring and treatments, baseline and follow-up thyroid function (serum levels of FT3, FT4, and TSH) and morning ACTH and cortisol are crucial, because imbalance of hypothalamuspituitary-thyroid (H-P-T) axis or hypothalamuspituitary-adrenal (H-P-A) axis is life-threating. A cosyntropin (; synthetic ACTH), infusion test is helpful to distinguish primary or secondary (pituitary-originated) adrenal insufficiency. Levels of GH-IGF-1, gonadotropin-sex hormones, and prolactin, should be assessed in patients with hypophysitis or hypopituitarism. High-dose steroids (e.g. $1-2 \mathrm{mg} / \mathrm{kg} /$ day of oral prednisolone) is used as treatment for those patients with severe headache, or visual abnormalities ${ }^{[15,16]}$. In cases of critical hypophysitis or hypopituitarism, intravenous glucocorticoid infusion is considered. Otherwise, oral hydrocortisone $(15-30 \mathrm{mg} /$ day $)$ is used for treatment of adrenal insufficiency. In cases of adrenal crisis, higher dose of hydrocortisone $(300 \mathrm{mg} /$ day $)$ is administrated intravenously. Levothyroxine (L-T4) (typically starting from $25 \mu \mathrm{g} /$ day with increasing dosage) is used for secondary hypothyroidism; but adrenal insufficiency should be treated first to avoid adrenal crisis induced by increasing thyroid hormone effect. In cases of secondary hypogonadism or GH insufficiency, gonadotropin replacement or $\mathrm{GH}$ replacement therapy can be considered, respectively. In cases of central diabetes insipidus, Desmopressin acetate (DDAVP) is used ${ }^{[17]}$.

\section{Thyroiditis related to ir $\mathrm{AE}$}

The thyroid gland is an organ to produce thyroid hormone (T3, T4), and controls systemic metabolism. Major autoimmune thyroid diseases (AITD)s are Graves' disease (GD) in which TSH receptor is autoantigen (20), and Hashimoto's thyroiditis (HT) in which TPO and Tg are autoantigen ${ }^{[21]}$. Cellmediated immunity and antibody-dependent cellular cytotoxicity (ADCC) are involved in AITD. An interim analysis of Japanese patients with malignant melanoma underwent ICI therapy showed high prevalence of thyroid disorders (any grade; $23.53 \%$, and $\geq$ Grade $3 ; 0.44 \%)^{[22]}$. In contrast to pituitary irAEs, the thyroid gland is preferentially affected by antiPD-1 antibody rather than anti-CTLA-4 antibody. Thyroiditis related to irAE occurs in $0-4 \%$ patients treated with anti-CTLA-4 antibody ${ }^{[7]}$. Prevalence of thyroiditis by anti-PD-1 antibodies is estimated to be $9 \%{ }^{[6]}$. IrAE-associated thyrotoxicosis and hypothyroidism similarly prevalent ${ }^{[6,23,24]}$. Transient thyrotoxicosis may precede hypothyroidism ${ }^{[23,24]}$. Dual treatment of nivolumab and ipilimumab induces thyroiditis in $22 \%$ of patients ${ }^{[25]}$. More recent reports showed increased incidence of anti-PD-1 antibodyinduced thyroid dysfunction in $17.1 \%$ of malignant melanoma patients ${ }^{[24]}$ and $21 \%$ of non-small cell lung cell cancer patients, respectively ${ }^{[26]}$. Morganstein, et al reported that ipilimumab-induced thyroid dysfunction occurred in $23 \%$ of patients, anti-PD1 -induced thyroid dysfunction in $39.1 \%$ of those, and dual treatment induced thyroid dysfunction in $50 \%$ of those ${ }^{[27]}$. Anti-PD-L1 antibody treatment was reported to induce $2-4 \%$ of thyroid dysfunction ${ }^{[28]}$. Patients who have anti-TPO-antibody (TPOAb) or anti-Tg-antibody ( $\mathrm{TgAb})$ are reported to be prone for development of thyroiditis related to irAE ${ }^{[29]}$. Thyroiditis related to irAE can occur early; between 2 and 6 weeks and mostly within 6 months after treatment. Notably, a proportion of the ICI-treated patients exhibit 'non-thyroidal illness syndrome' (normal FT4, normal TSH, and decreased FT3), reflecting lower nutrition status. Thyroid dysfunction during pembrolizumab treatment was reported to have possible association with feasible outcome of lung cancer ${ }^{[29]}$.

Thyrotoxicosis: thyrotoxicosis related to irAE is classified into two subtypes; 1) destructive thyroiditis, and 2) hyperthyroidism manifested as GD. Palpitation, weight loss, tremor, fatigue, and diarrhea are typically seen as thyrotoxic symptoms.

1) Destructive thyroiditis: destructive thyroiditis is often induced by ICI, transient increase of FT3 and FT4 are seen, concomitant with suppressed TSH levels. Anti-TSH receptor antibody (TRAb) is negative in most of the cases. TPOAb or $\mathrm{TgAb}$ are often positive at diagnosis ${ }^{[23,24]}$. Elevation of serum $\mathrm{Tg}$ levels may help the diagnosis of thyroiditis ${ }^{[30]}$. In thyroid ultrasonography, Doppler blood flow is not increased, thyroid uptake in ${ }^{123} \mathrm{I}$ or ${ }^{99 \mathrm{~m}} \mathrm{Tc}$ scintigram is usually decreased, and FDG-PET is positively accumulated in the thyroid ${ }^{[24]}$. Diffuse thyroid enlargement can be seen ${ }^{[29]}$. In treatment, $\beta$-blocker is effective for relieving thyrotoxic symptoms. Effectivity of steroid treatment is not clear.

2) GD induced by ICI: Rare cases of thyrotoxicosis show elevated FT3 and FT4 concomitant with suppressed TSH, and positivity of TRAb, suggesting GD during ICI treatment ${ }^{[31]}$. In those cases, increased color Doppler flow and increased uptake in ${ }^{123} \mathrm{I}$ or ${ }^{99 \mathrm{~m}} \mathrm{Tc}$ scintigram were seen, 
and treatment options are anti-thyroid drug, ${ }^{131} \mathrm{I}$ treatment, or thyroid surgery.

Hypothyroidism: Hypothyroidism related to irAE is classified into two subtypes; 1) transient thyrotoxicosis followed by hypothyroidism, and 2) a single phase of hypothyroidism ${ }^{[6,23,24]}$. Hypothyroidism is due to thyroiditis by ICI. Patients who have $\mathrm{TgAb}$ or TPOAb are prone to be hypothyroidism ${ }^{[29]}$. Primary hypothyroidism is diagnosed by decreased FT3 and FT4, and increased TSH. Primary hypothyroid symptoms are similar to those in secondary hypothyroidism mentioned above. In thyroid ultrasonography, Doppler blood flow may be decreased, and thyroid uptake in ${ }^{123} \mathrm{I}$ or ${ }^{99 \mathrm{~m}} \mathrm{Tc}$ scintigram is decreased. Diffuse thyroid swelling is seen in acute phase of thyroiditis, and atrophy can be seen in subsequent hypothyroid phase ${ }^{[29]}$. L-T4 (typically starting from $25 \mu \mathrm{g}$ /day with increasing dosage) is used to treat hypothyroidism. Cases of hypothyroidism often progress to permanent hypothyroidism ${ }^{[23]}$. In monitoring, baseline measurement of thyroid autoantibodies (TRAb, $\mathrm{TgAb}$, and TPOAb), and routine checkup of serum FT3, FT4 and TSH levels are recommended. Eosinophilia, thrombocytopenia, ESR/CRP/LDH elevation, and liver dysfunction might be important for earlier detection of thyrotoxicosis ${ }^{[9]}$.

\section{Adrenalitis related to ir $\mathrm{AE}$}

Adrenal glands secrete adrenal hormones to maintain systemic activity. Cortisol is one of the critical adrenal hormones, regulating glucose metabolism, blood pressure, and other homeostasis. Cases of autoimmune adrenalitis were reported, with presence of autoantibodies to $\mathrm{P} 450 \mathrm{c} 21$ or P450c17; adrenal enzyme involved with adrenal hormone synthesis ${ }^{[32]}$. Adrenal failure is due to incomplete secretion of adrenal hormones including cortisol. IrAE-associated adrenal hypofunction is classified to primary or secondary adrenocortical insufficiency. Primary adrenocortical insufficiency is due to adrenalitis ${ }^{[10]}$, and secondary adrenocortical insufficiency is due to pituitary damage described above $^{[5]}$. Prevalence of adrenalitis related to irAE is reported as approximately $1 \%{ }^{[33]}$. Adrenal dysfunction usually occurs within 1-6 months after initiation of $\mathrm{ICI}^{[10,34-36]}$. Either anti-PD-1 antibody ${ }^{[10,34]}$ or anti-CTLA-4 antibody ${ }^{[35,36]}$ induces adrenalitis.

Symptoms in primary adrenal insufficiency are almost the same as those seen in secondary adrenal insufficiency. Decreased serum cortisol, increased plasma ACTH, and decreased urine cortisol levels indicate diagnosis of adrenalitis. In an ACTH stimulation test, response of cortisol is lost in cases of primary adrenal insufficiency. Presence of autoantibodies to P450c21 or P450c17 may be useful for diagnosis of adrenalitis. As a treatment, oral hydrocortisone replacement therapy $(15-30 \mathrm{mg} /$ day $)$ is used. In cases of adrenocortical crisis, higher dose of hydrocortisone $(300 \mathrm{mg} /$ day $)$ are administrated intravenously.

\section{Type 1 diabetes mellitus and other endocrine dysfunctions related to ir $\mathrm{AE}$}

Pancreatic beta cells produce and secrete insulin. Insulin controls blood glucose levels. In patients with type 1 diabetes (T1DM), pancreatic beta cells are destroyed, and insulin secretion is attenuated. Insulin is considered to be an autoantigen in T1DM. As a pancreatic irAE, patients with T1DM during ICI therapy were reported ${ }^{[11,12,37]}$. Prevalence of T1DM associated with ICI therapy was reported to be $0.6 \%{ }^{[37]}$. Anti-PD-1 antibodies more often induce T1DM than anti-CTLA-4 antibodies. As a severe subtype of T1DM, fulminant type 1 diabetes (F1DM) was also reported. T1DM related to irAE occurs mostly within 1-6 months after ICI treatment, and often rapidly progressing with ketoacidosis. T1DM related to irAE is thus one of the urgent complications of irAE.

Thirst, polydipsia, and polyuria are seen as hyperglycemic symptoms, and elevated plasma glucose is seen. HbA1c levels are not always elevated, reflecting acute development of hyperglycemia. Sometimes swelling of pancreas is seen in abdominal CT. Anti-GAD, anti-IA2, and anti-insulin autoantibodies are sometimes positive. Immediate insulin therapy is required in patients with T1DM related to irAE. Monitoring of plasma glucose and $\mathrm{HbA} 1 \mathrm{c}$ are useful for detection of T1DM. Measurement of anti-GAD antibody is also helpful to find individuals who may be susceptible to T1DM. Although rare, hypoparathyroidism related to irAE was reported as an endocrine irAE ${ }^{[13]}$.

\section{Possible mechanisms in endocrine irAEs}

Multiple factors influence in the development of endocrine-related irAEs. In certain genetic and environment backgrounds, ICI may activate immune systems especially autoreactive cytotoxic $\mathrm{CD} 8^{+}$ $\mathrm{T}$-cells directed to endocrine organs ${ }^{[2]}$. 
Genetic factors of immune-checkpoints in irAEs

Altered function or genetic mutation of immunecheckpoint molecules may contribute to the development of endocrine irAEs. In AITD, genetic mutations in CLTA-4 in $\mathrm{GD}^{[38]}$ and $\mathrm{HT}^{[39]}$ were reported. An association of a SNP in PD-L1 with GD was shown ${ }^{[40]}$. SNPs in CLTA-4 and PD-L1 also contribute to the development of autoimmune adrenalitis ${ }^{[41]}$. PD-L1 and PD-1 genetic mutation are reported to be involved in T1DM development ${ }^{[42,43]}$.

\section{Molecular mimicry of tumor antigen and auto- antigen in endocrine organs}

One of the tumor-associated antigen: NY-ESO-1 possess common amino acid sequence with thyroid autoantigens (TSH receptor, $\mathrm{Tg}$, and TPO), and administration of NY-ESO-1 was reported to induce GD or HT in individuals who have risk allele of MHC (class I or class II) ${ }^{[44]}$. In a case of malignant melanoma with F1DM during ICI treatment, HLADR4 restricted insulin autoantigen with positive conversion of anti-insulin antibody was suggested ${ }^{[11]}$. Therefore, upon both activation of anti-pancreatic beta cells and anti-melanoma immunity, exacerbation of pancreatic beta cell function and improvement of malignant melanoma can be seen in patients with F1DM related to irAE. Distinct manifestations of irAE inpatients with various malignant diseases were reported (e.g. malignant melanoma patients had a higher frequency of gastrointestinal/skin irAE, and a lower frequency of pneumonitis related to irAE) ${ }^{[45]}$. Thus, various tumor epitope may possess common amino acid sequences with endocrine epitope (e.g. TSH receptor, Tg, TPO, insulin). And crosspresentation of those on HLA molecules may be related to endocrine irAEs (Figure 1,2).

\section{Inhibition the role of immune-checkpoints in endocrine irAEs}

Anti-CTLA-4 antibodies often induce autoimmunity, by inhibiting regulatory T-cells function, and by activation of immunogenic $\mathrm{CD} 4{ }^{+} \mathrm{T}$-cells and $\mathrm{CD}^{+}{ }^{+}$-cells ${ }^{[46,47]}$. In animal models, anti-CTLA-4antibodies were reported to induce thyroiditis ${ }^{[48]}$, adrenalitis $^{[49]}$, and T1DM ${ }^{[50]}$. Anti-PD-1 antibodies blocks PD-1 pathway which suppress autoimmunity. Subsequently, immunogenic $\mathrm{CD} 4^{+} \mathrm{T}$-cells and CD8 ${ }^{+}$T-cells can be activated. PD-1 knockout mice showed lupus-like autoimmune disorders ${ }^{[2]}$, and PD-1 blockade was reported to induce T1DM in mice $^{[51]}$.

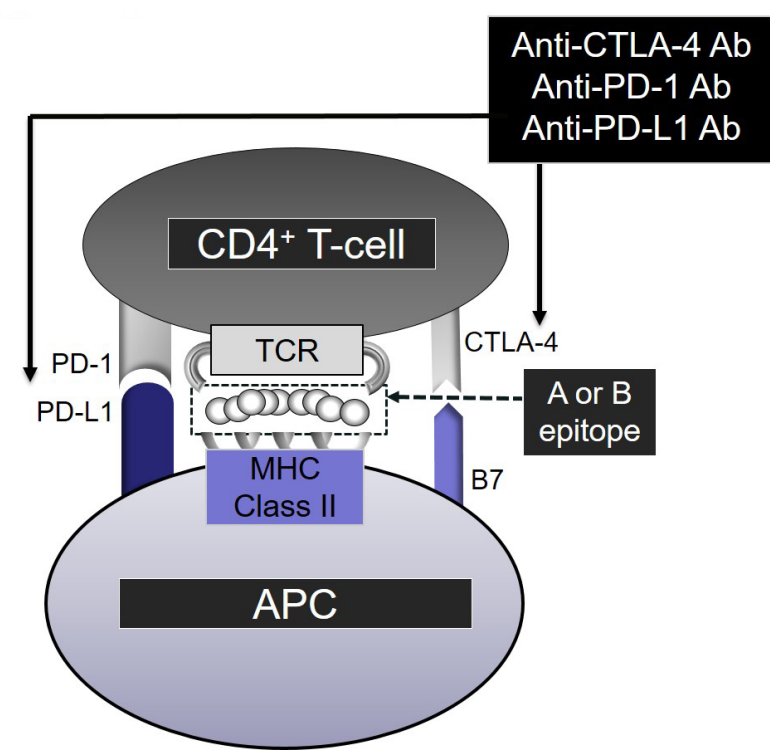

Figure 2. Relation of immune-checkpoints and $\mathrm{CD} 4^{+}$ T-cell is shown. A or B epitopes are presented on the surface of APC with MHC-class II to TCR on CD4 $4^{+}$T-cell. Immune-checkpoint inhibitors regulate these immune reaction, and $\mathrm{CD}^{+} \mathrm{T}$-cell is activated.

A: tumor antigen, $\mathrm{B}$ endocrine organs specific antigens (e.g. thyroglobulin, insulin). APC: antigen presenting cell; TCR: T-cell receptor

\section{Other possible mechanisms in endocrine irAEs}

Higher expression of CTLA-4 in the pituitary gland was reported to be related to hypophysitis, due to type II or type IV allergy by IgG1 or IgG2 type anti-CTLA-4 antibodies ${ }^{[18]}$. Yamauchi, et al. reported that PD-L1 and PD-L2 expressions on thyroid gland might be related to nivolumab-induced thyroiditis ${ }^{[52]}$.

\section{Discussion}

To date, most authors have recommended both discontinuation of ICIs and high dose glucocorticoid for treatment of irAEs. However, this has been challenged by recently, particularly if the endocrine irAEs can be managed, and the anti-tumor therapy is effective ${ }^{[53]}$. In addition, correlation of prevalence of irAEs to anti-tumor immunity was recently shown $^{[54]}$. It is of interest to examine whether biomarkers which have been reported in malignant melanoma can be applied to the patients with endocrine irAEs ${ }^{[55]}$. Further investigations with larger number of cases over longer periods are warranted to establish diagnostic and therapeutic approaches to endocrine irAEs. In the future, identification of individuals (e.g. genetic predispositions such as HLA type, age, gender) who are susceptible to irAEs are necessary ${ }^{[3,4]}$. 


\section{Conflict of interest}

The authors declare no potential conflict of interest with respect to the research, authorship, and/or publication of their article.

\section{References}

1. Blank CU, Enk A. Therapeutic use of anti-CTLA-4 antibodies. Int Immunol 2015; 27(1): 3-10. doi: 10.1093/intimm/dxu076.

2. Okazaki T, Honjo T. PD-1 and PD-1 ligands: From discovery to clinical application. Int Immunol 2007; 19(7): 813-824. doi: 10.1093/intimm/ dxm057

3. Hodi FS, O'Day SJ, McDermott DF, et al. Improved survival with ipilimumab in patients with metastatic melanoma. N Engl J Med 2010; 363(8): 711-723. doi: 10.1056/NEJMoa1003466.

4. Topalian SL, Hodi FS, Brahmer JR, et al. Safety, activity, and immune correlates of anti-PD-1 antibody in cancer. N Engl J Med 2012; 366(26): 2443-2454. doi: 10.1056/NEJMoa1200690.

5. Byun DJ, Wolchok JD, Rosenberg LM, et al. Cancer immunotherapy - immune checkpoint blockade and associated endocrinopathies. Nat Rev Endocrinol 2017; 13(4): 195-207. doi: 10.1038/ nrendo.2016.205.

6. Torino F, Corsello SM, Salvatori R. Endocrinological side-effects of immune checkpoint inhibitors. Curr Opin Oncol 2016; 28(4): 278-287. doi: 10.1097/ CCO.0000000000000293.

7. Corsello SM, Barnabei A, Marchetti P, et al. Endocrine side effects induced by immune checkpoint inhibitors. J Clin Endocrinol Metab 2013; 98(4): 1361-1375. doi: 10.1210/jc.20124075.

8. Weber JS, D'Angelo SP, Minor D, et al. Nivolumab versus chemotherapy in patients with advanced melanoma who progressed after anti-CTLA-4 treatment (CheckMate 037): A randomised, controlled, open-label, phase 3 trial. Lancet Oncol 2015; 16(4): 375-384. doi: 10.1016/S14702045(15)70076-8.

9. Ariyasu $\mathrm{H}$, Inaba $\mathrm{H}$, Ota $\mathrm{T}$, et al. Thyrotoxicosis and Adrenocortical Hormone Deficiency During Immune-checkpoint Inhibitor Treatment for Malignant Melanoma. In Vivo 2018; 32(2): 345351. doi: 10.21873/invivo.11244

10. Trainer H, Hulse P, Higham CE, et al. Hyponatraemia secondary to nivolumab-induced primary adrenal failure. Endocrinol Diabetes Metab Case Rep 2016; 2016. pii: 16-0108 doi: 10.1530/EDM-16-0108

11. Shiba M, Inaba $\mathrm{H}$, Ariyasu $\mathrm{H}$, et al. A case of fulminant type 1 diabetes mellitus accompanied by positive conversion of anti-insulin antibody after the administration of anti-CTLA-4 antibody following the discontinuation of anti-PD-1 antibody. Intern Med 2018; Feb 28. doi: 10.2169/ internalmedicine.9518-17.

12. Teló GH, Carvalhal GF, Cauduro CGS, et al. Fulminant type 1 diabetes caused by dual immune checkpoint blockade in metastatic renal cell carcinoma. Ann Oncol 2017; 28(1): 191-192. doi: 10.1093/annonc/mdw447.

13. Win MA, Thein KZ, Qdaisat A, et al. Acute symptomatic hypocalcemia from immune checkpoint therapy-induced hypoparathyroidism. Am J Emerg Med 2017; 35(7): 1039.e5-1039.e7. doi: 10.1016/j.ajem.2017.02.048.

14. Caturegli P, Newschaffer C, Olivi A, et al. Autoimmune hypophysitis. Endocr Rev 2005; 26(5): 599-614. doi: 10.1210/er.2004-0011

15. Faje A. Immunotherapy and hypophysitis: clinical presentation, treatment, and biologic insights. Pituitary 2016; 19(1): 82-92. doi: 10.1007/s11102015-0671-4.

16. Faje AT, Sullivan R, Lawrence D, et al. Ipilimumab-induced hypophysitis: a detailed longitudinal analysis in a large cohort of patients with metastatic melanoma. J Clin Endocrinol Metab 2014; 99(11): 4078-4085. doi: 10.1210/ jc.2014-2306.

17. Zhao C, Tella SH, Del Rivero J, et al. Anti-PD-L1 Treatment induced central diabetes insipidus. J Clin Endocrinol Metab 2018; 103(2): 365-369. doi: 10.1210/jc.2017-01905.

18. Caturegli P, Di Dalmazi G, Lombardi M, et al. Hypophysitis secondary to cytotoxic T-lymphocyte-associated protein 4 blockade: Insights into pathogenesis from an autopsy series. Am J Pathol 2016; 186(12): 3225-3235. doi: 10.1016/j.ajpath.2016.08.020.

19. Iwama S, De Remigis A, Callahan MK, et al. Pituitary expression of CTLA-4 mediates hypophysitis secondary to administration of CTLA4 blocking antibody. Sci Transl Med 2014; 6(230): 230ra45. doi: 10.1126/scitranslmed.3008002.

20. Akamizu T, Mori T, Nakao K. Pathogenesis of Graves' disease: Molecular analysis of antithyrotropin receptor antibodies. Endocr J 1997; 44(5): 633-646. PMID: 9466318

21. Akamizu T, Amino N. Hashimoto's Thyroiditis. In: De Groot LJ, Chrousos G, Dungan K, Feingold $\mathrm{KR}$, et al. editors. Endotext [Internet]. South Dartmouth (MA): MDText.com, Inc.; 20002017 Jul 17. PMID: 25905412 Bookshelf ID: NBK285557

22. Kiyohara Y, Uhara H, Ito Y, et al. Safety and efficacy of nivolumab in Japanese patients with malignant melanoma: An interim analysis of a postmarketing surveillance. J Dermatol 2018; 45(4): 408-415. doi: 10.1111/1346-8138.14227.

23. Orlov S, Salari F, Kashat L, et al. Induction of painless thyroiditis in patients receiving programmed death 1 receptor immunotherapy for metastatic malignancies. J Clin Endocrinol Metab 2015; 100(5): 1738-1741. doi: 10.1210/jc.20144560 . 
24. de Filette J, Jansen Y, Schreuer M, et al. Incidence of Thyroid-Related Adverse Events in Melanoma Patients Treated With Pembrolizumab. J Clin Endocrinol Metab 2016; 101(11): 4431-4439. doi: 10.1210/jc.2016-2300

25. Ryder M, Callahan M, Postow MA, et al. Endocrine-related adverse events following ipilimumab in patients with advanced melanoma: a comprehensive retrospective review from a single institution. Endocr Relat Cancer 2014; 21(2): 371381. doi: 10.1530/ERC-13-0499.

26. Osorio JC, Ni A, Chaft J, et al. Antibody-mediated thyroid dysfunction during T-cell checkpoint blockade in patients with non-small-cell lung cancer. Ann Oncol 2017; 28(3): 583-589. doi: 10.1093/annonc/mdw640.

27. Morganstein DL, Lai Z, Spain L, et al. Thyroid abnormalities following the use of cytotoxic T-lymphocyte antigen-4 and programmed death receptor protein-1 inhibitors in the treatment of melanoma. Clin Endocrinol 2017; 86(4): 614-620. doi: $10.1111 /$ cen.13297.

28. Torino F, Corsello S, Salvatori R, et al. Endocrinological side-effects of immune checkpoint inhibitors. Curr Opin Oncol 2016; 28(4): 278-287. doi: 10.1097/CCO.0000000000000293.

29. Kobayashi T, Iwama S, Yasuda Y, et al. Patients With Antithyroid Antibodies Are Prone To Develop Destructive Thyroiditis by Nivolumab: A Prospective Study. J Endocr Soc 2018; 2(3): 241-251. doi. 10.1210/js.2017-00432

30. Pearce EN, Farwell AP, Braverman LE. Thyroiditis. N Engl J Med 2003; 348(26): 2646-2655. DOI: 10.1056/NEJMra021194

31. Gan EH, Mitchell AL, Plummer R, et al. Tremelimumab-Induced Graves Hyperthyroidism. Eur Thyroid J 2017; 6(3): 167-170. doi: $10.1159 / 000464285$.

32. Brandão Neto RA, de Carvalho JF. Diagnosis and classification of Addison's disease (autoimmune adrenalitis). Autoimmun Rev 2014; 13(4-5): 40811. doi: 10.1016/j.autrev.2014.01.025.

33. Joshi MN, Whitelaw BC, Palomar MT, et al. Immune checkpoint inhibitor-related hypophysitis and endocrine dysfunction: clinical review. Clin Endocrinol 2016;85(3):331-339. doi: 10.1111/ cen. 13063.

34. Paepegaey AC, Lheure C, Ratour C, et al. Polyendocrinopathy resulting from pembrolizumab in a patient with a malignant melanoma. J Endocr Soc 2017; 1(6): 646-649. doi: 10.1210/js.2017-00170.

35. Min L, Ibrahim N. Ipilimumab-induced autoimmune adrenalitis. Lancet Diabetes Endocrinol 2013; 1(3): e15. doi: 10.1016/S22138587(13)70031-7.

36. Bacanovic S, Burger IA, Stolzmann P. Ipilimumabinduced adrenalitis: A possible pitfall in 18F-FDGPET/CT. Clin Nucl Med 2015; 40(11): e518-519. doi: 10.1097/RLU.0000000000000887.
37. Scott ES, Long GV, Guminski A, et al. The spectrum, incidence, kinetics and management of endocrinopathies with immune checkpoint inhibitors for metastatic melanoma. Eur J Endocrinol 2018; 178(2): 175-182. doi: 10.1530/ EJE-17-0810.

38. Akamizu T, Sale M, Rich S, et al. Association of autoimmune thyroid disease with microsatellite markers for the thyrotropin receptor gene and CTLA4 in Japanese patients. Thyroid 2000; 10(10): 851858. doi: 10.1089/thy.2000.10.851

39. Bicek A, Zaletel K, Gaberscek S, et al. 49A/ $\mathrm{G}$ and CT60 polymorphisms of the cytotoxic T-lymphocyte-associated antigen 4 gene associated with autoimmune thyroid disease. Hum Immunol 2009; 70(10): 820-824. doi: 10.1016/ j.humimm.2009.06.016.

40. Hayashi M, Kouki T, Takasu N, et al. Association of an $\mathrm{A} / \mathrm{C}$ single nucleotide polymorphism in programmed cell death-ligand 1 gene with Graves' disease in Japanese patients. Eur J Endocrinol 2008; 158(6): 817-822. doi: 10.1530/EJE-07-0649.

41. Falorni A, Brozzetti A, Perniola R. From genetic predisposition to molecular mechanisms of autoimmune primary adrenal insufficiency. Front Horm Res 2016; 46: 115-132. doi: 10.1159/000443871.

42. Pociot F, Lernmark Å. Genetic risk factors for type 1 diabetes. Lancet 2016; 387(10035): 2331-2339. doi: 10.1016/S0140-6736(16)30582-7.

43. Pizarro C, García-Díaz DF, Codner E, et al. PDL1 gene polymorphisms and low serum level of PD-L1 protein are associated to type 1 diabetes in Chile. Diabetes Metab Res Rev 2014; 30(8): 761-766. doi: 10.1002/dmrr.2552.

44. Vita R, Guarneri F, Agah R, et al. Autoimmune thyroid disease elicited by NY-ESO-1 vaccination. Thyroid 2014; 24(2): 390-394. doi: 10.1089/ thy.2013.0170.

45. Khoja L, Day D, Wei-Wu Chen T, et al. Tumourand class-specific patterns of immune-related adverse events of immune checkpoint inhibitors: A systematic review. Ann Oncol 2017; 28(10): 2377-2385. doi: 10.1093/annonc/mdx286.

46. Weber JS, Hamid O, Chasalow SD, et al. Ipilimumab increases activated $\mathrm{T}$ cells and enhances humoral immunity in patients with advanced melanoma. J Immunother 2012; 35(1): 89-97. doi: 10.1097/ CJI.0b013e31823aa41c.

47. Simpson TR, Li F, Montalvo-Ortiz W, et al. Fcdependent depletion of tumor-infiltrating regulatory $\mathrm{T}$ cells co-defines the efficacy of anti-CTLA-4 therapy against melanoma. J Exp Med 2013; 210(9): 1695-1710. doi: 10.1084/jem.20130579.

48. Kari S, Flynn JC, Zulfiqar M, et al. Enhanced autoimmunity associated with induction of tumor immunity in thyroiditis-susceptible mice. Thyroid 2013; 23(12): 1590-1599. doi: 10.1089/ thy.2013.0064. 
49. Vudattu NK, Waldron-Lynch F, Truman LA, et al. Humanized mice as a model for aberrant responses in human T cell immunotherapy. J Immunol 2014; 193(2): 587-596. doi: 10.4049/jimmunol.1302455.

50. Ansari MJ, Salama AD, Chitnis T, et al. The programmed death-1 (PD-1) pathway regulates autoimmune diabetes in nonobese diabetic (NOD) mice. J Exp Med 2003; 198(1): 63-69.

51. Kochupurakkal NM, Kruger AJ, Tripathi S, et al. Blockade of the programmed death-1 (PD1) pathway undermines potent genetic protection from type 1 diabetes. PLoS One 2014; 9(2): e89561. doi: 10.1371/journal.pone.0089561

52. Yamauchi I, Sakane Y, Fukuda Y, et al. Clinical features of nivolumab-induced thyroiditis: A case series study. Thyroid 2017; 27(7): 894-901. doi: 10.1089/thy.2016.0562
53. Brahmer JR, Lacchetti C, Schneider BJ, et al. Management of immune-related adverse events in patients treated with immune checkpoint inhibitor therapy: American Society of Clinical Oncology Clinical Practice Guideline. J Clin Oncol 2018: JCO2017776385. doi: 10.1200/JCO.2017.77.6385

54. Sato K, Akamatsu H, Murakami E, et al. Correlation between immune-related adverse events and efficacy in non-small cell lung cancer treated with nivolumab. Lung Cancer 2018; 115 : 71-74. doi: 10.1016/j.lungcan.2017.11.019.

55. Seidel JA, Otsuka A, Kabashima K. Treating tumors with immune checkpoint inhibitors: Rationale and limitations. Trends Immunother 2017; 1(1): 2-9. doi: 10.24294/ti.v1.i1.20 